\title{
Flow Shop Scheduling Using Genetic Algorithm
}

\author{
Vinoj K \\ Asst. professor, Mechanical Engineering, \\ Viswajyothi College of Engineering \& Technology, Muvattupuzha, Kerala, India \\ Tijo Jose \\ Asst. professor, Mechanical Engineering, \\ Viswajyothi College of Engineering \& Technology, Muvattupuzha Kerala, India
}

\begin{abstract}
A flow shop is a production system in which machines are arranged in the order in which operations are performed on jobs. The flow shop is characterized by a flow of work that is unidirectional. The problem of scheduling in a flow-shop is considered in the present work with the objective of minimizing mean flow time. Mean flow time is average of the completion time of all jobs. In the present work, genetic algorithm (GA) is used for the solution of the flow shop scheduling problem. Small size and large size problems are considered for the experiments. The performance of a genetic algorithm depends very much on the selection of the proper genetic operators. The various genetic operators such as single-point crossover with shift mutation, single-point crossover with random exchange mutation, two-point crossover with shift mutation, two-point crossover with random exchange mutation are examined. It is found that GA with twopoint crossover and shift mutation operator provides better solutions in many of the problems considered in the present work.
\end{abstract}

Keywords- Mean flow time, genetic algorithm, single-point crossover, shift mutation

\section{INTRODUCTION}

Scheduling is considered to be the major function for shop floor productivity improvement. Scheduling is the allocation of resources applying the limiting factors of time and cost to perform a collection of tasks [1]. A flow shop is a manufacturing system in which machines are arranged in the order in which operations are performed on jobs. The flow shop is characterized by a flow of work that is unidirectional. Flow shop scheduling problem deals with the ordering of processing jobs, where each job is to be processed in the identical ordering of machines or stages. The flow shop scheduling problem can be defined as follows [2]. Given ' $\mathrm{n}$ ' jobs to be processed on ' $\mathrm{m}$ ' machines in the same technological order, the processing time of job ' $i$ ' on machine ' $\mathrm{j}$ ' being $\mathrm{P}_{\mathrm{ij}}(\mathrm{i}=1,2,3, \ldots \ldots \ldots . . \mathrm{n}$ and $\mathrm{J}=1,2, \ldots \ldots \ldots . \mathrm{m}$ ); it is desired to find the order (sequence) in which the ' $\mathrm{n}$ ' jobs should be processed on each of the ' $\mathrm{m}$ ' machines to minimize a well defined measure of performance or objective function. In the present paper, the objective function considered is mean flow time. Flow time is the amount of time spent by a job in the system. Flow time of a job is computed as the difference between its completion time and its ready time. When all jobs to be scheduled are available at time zero, the flow time of a job is its completion time. Flow time has a direct relationship with the work-in-process inventory and hence a schedule with minimum mean flow time results in minimum workin-process inventory.

Johnson's algorithm [2] provides optimal solution for two-machine, n-job flow shop scheduling problem with makespan as the criterion. For larger-size problems, heuristic algorithms are generally used $[3,4,5]$. Reeves [6] presents a genetic algorithm (GA) for flow shop scheduling with makespan as the performance criterion. The performance of GA depends very much on the selection of proper genetic operators. Crossover and mutation are the two major operators in any GA. The present study investigates the impact of various genetic operators on the genetic search by conducting computational experiments on the flow-shop scheduling problem (FSSP). The rest of the paper is organized as follows. Section 2 provides the salient features of the GA approach. The details of the GA applied 
for the flow shop scheduling problem are presented in section 3. The experimentation carried out is explained in section 4. Results and discussion are provided in section 5. Conclusions are presented in section 6.

\section{GENETIC ALGORITHM APPROACH}

Genetic algorithms [7] are computerized iterative search optimization algorithms based on the mechanics of natural genetics and natural selection. They combine survival of the fittest among the string structures with a structured yet randomized information exchange to form a search algorithm. GAs encode a potential solution to a specific problem as a simple chromosome like data structure and apply recombination operators to these structures to preserve critical information. An implementation of genetic algorithm begins with an initial population of typically random chromosomes. One then evaluates these structures and allocates reproductive opportunities in such a way that those chromosomes, which represent a better solution to the target problem, are given more chances to reproduce than those chromosomes, which represent poorer solutions. The goodness of a solution is typically defined with respect to the current population.

As pointed earlier, GA's mimic the survival of the fittest principle of nature to make a search process. Therefore, GA's are naturally suitable for solving maximization problems. Minimization problems are usually transformed into maximization problems by some suitable transformation. In general, a fitness function $\mathrm{F}(\mathrm{x})$ is first derived from the objective function and used in successive genetic operations. For maximization problems, the fitness function can be considered to be the same as the objective function or $F(x)=f(x)$. For minimization problems, the fitness function is an equivalent maximization problem chosen such that optimum point remains unchanged. A number of such transformations are possible. The following fitness function is often used

$$
\mathbf{F}(\mathbf{x})=\mathbf{1} /(\mathbf{1}+\mathbf{f}(\mathbf{x}))
$$

Here, $\mathrm{f}(\mathrm{x})=$ objective function value. This transformation does not alter location of the minimum, but converts a minimization problem to an equivalent maximization problem. The fitness function of a string is known as the string's fitness.

\subsection{GA OPERATORS}

The application of GA begins with a population of random strings representing design or decision variables. Therefore, each string is evaluated to find the fitness value. The population is then operated by three main parameters - reproduction, crossover and mutation to create a new population of points. The new population is further evaluated and tested for termination. If the termination criteria is not met, the population is iteratively operated by the above three operators and evaluated. This procedure is continued until the termination criterion is met. One cycle of these operations and the subsequent evaluation procedure is known as a generation in GA's terminology.

\section{GA FOR FLOW SHOP SCHEDULING PROBLEM}

In the present study, GA is applied for solving the flow shop scheduling problem with minimization of mean flow time as the objective. The following are the assumptions made:

- All the $\mathrm{n}$ jobs are processed on all the $m$ machines in the same order.

- All jobs and machines are available at the beginning of the scheduling period, i.e., at time zero.

- Each job is processed only once on each machine.

- Each job requires a known and finite processing time and is independent of the order in which jobs are processed.

- No pre-emption is allowed i.e., an operation once started on a machine must be completed before another operation may be started on that machine.

- Machines never breakdown and are available throughout the scheduling period.

- In-process inventory is allowed i.e there is no restriction on the number of jobs waiting near a machine for processing.

The steps of GA are as follows: 
Step 1: Randomly generate $\mathrm{n}$ feasible sequences ( $\mathrm{n}$ is the population size).

Step 2. Evaluate each sequence using the fitness function.

Step 3. Apply reproduction.

Step 4. Perform crossover to generate offspring.

Step 5. Mutate some members.

Step 6. Implement replacement strategy

Step 7. Repeat steps from 2 to 6 until termination criterion is satisfied

\section{Step 1 INITIALIZATION}

Each gene represents the job to be processed. The chromosome or string represents the schedule generated. Natural representation is used for the scheduling problem. The genetic search process starts with a randomly generated set of chromosomes called the initial population.

\section{Step 2. Population Evaluation}

The objective function considered is mean flow time. The initial population is evaluated for mean flow time.

\section{Step 3 Reproduction or Selection}

Roulette wheel selection method is used in the present study. It allocates offspring strings using a roulette wheel with slots sized according to fitness. This is a way of choosing members from the population of chromosomes in a way that is proportional to their fitness.

\section{Step 4 Crossover Operation}

The procedure for crossover is as follows. Random numbers between 0 and 1 are generated each time and if that random number is less than crossover probability, then two chromosomes are selected for crossover. Expected number of crossovers is calculated as shown below.

Expected number of crossovers $=$ crossover probability $*$ population size $/ 2$.

The remaining population is filled up with the chromosomes having the best objective value from initial population. The crossover probability considered in the present study is 0.6 .

Both single-point and two-point cross operators are used.

Single-point crossover: This crossover operation is illustrated in Figure 1. One point is randomly selected from the chromosome of one parent. The set of jobs on one side (each side is chosen with the same probability) is inherited from one parent to the child, and the other jobs are placed in the order of their appearance in the other parent. In Figure 1, Jobs A, B and C are inherited from parent 1 to the child, and the other jobs (i.e. Jobs C, D, E, F and G) are placed in the order of their appearance in Parent 2.

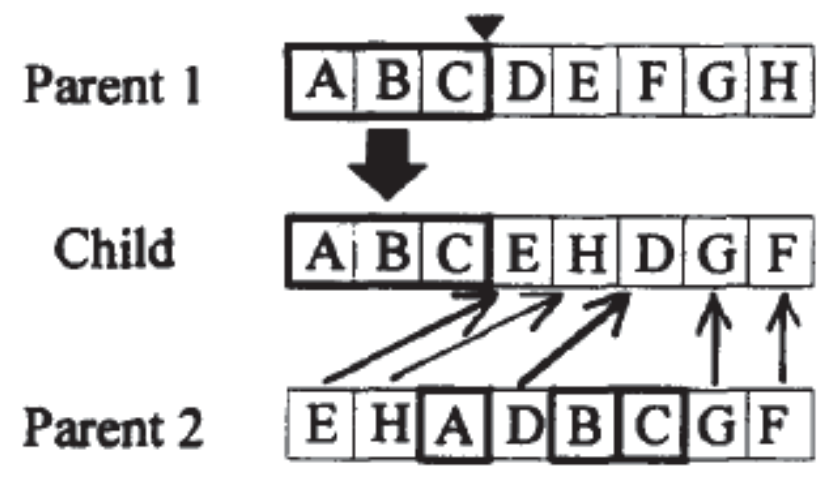

Figure 1 Single-Point Crossover

Two-point crossover: This crossover operation is illustrated in Figure 2. Two points are randomly selected from the chromosome of one parent. The jobs outside the selected two points are always inherited from one parent to the child, and the other jobs are placed in the same manner as the one-point crossover. 


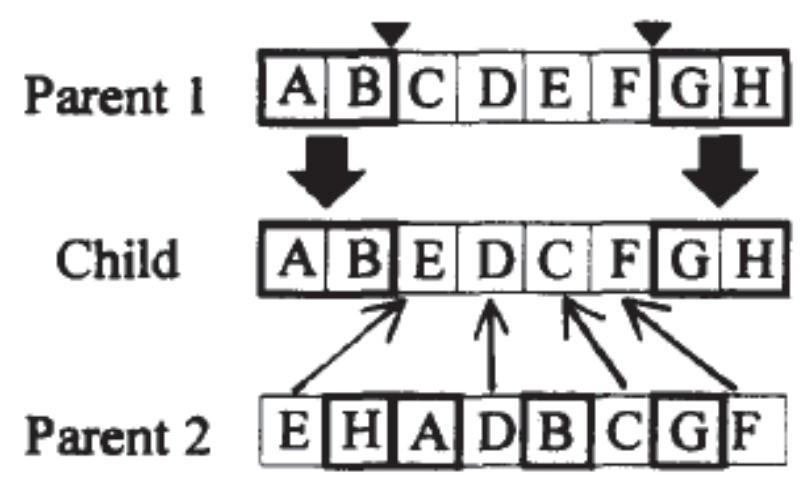

Figure 2 Two-point crossover

\section{Step 5 MUTATION}

Mutation is employed to give new information and reintroduce divergence into the population. This is a tool in avoiding local optima which is a common problem in stochastic algorithms. It also prevents the problem from becoming saturated with chromosomes that all look alike.

Mutation is applied on the population which is obtained from crossover. For this also, a random number between 0 and 1 is generated. If it is less than the mutation probability, then the chromosome will be selected for the mutation. This is repeated until the mutation rate is satisfied. Remaining chromosomes are copied from the crossover population. Mutation probability of 0.1 is considered in the present work. Two types of mutation are used in the present study. These are described as follows.

Random exchange mutation: Two positions are selected at random along the chromosome and the jobs contained in these positions are exchanged

\section{Random exchange mutation}

$\begin{array}{lllllllll}1 & 2 & 3 & 4 & 5 & 6 & 7 & 8 & 9 \\ 1 & 2 & 8 & 4 & 5 & 6 & 7 & 3 & 9\end{array}$

Shift mutation: A single job is selected randomly and inserted in a random position.

\begin{tabular}{lllllllll}
\multicolumn{10}{c}{ Shift mutation } \\
1 & 2 & 3 & 4 & 5 & 6 & 7 & 8 & 9 \\
1 & 6 & 2 & 3 & 4 & 5 & 7 & 8 & 9
\end{tabular}

Step 6 REPLACEMENT STRATEGY

The poor performing offsprings are replaced in the new generation with replacement strategy. The goal of the replacement strategy is to create generations of solutions that, on the average, outperform the previous generation. This is accomplished by restricting admittance to the new population to only those children that are better than members of current population.

In order to avoid strings which have more mean flow time value, the replacement strategy applied involves choosing best strings from mutation population and initial population.

Step 7 Termination

In the present work, the termination criterion used is as follows. If there is no improvement in the best solution found for a pre-specified number of generations, the algorithm terminates or if the algorithm reaches maximum number of generations the algorithm terminates.

\section{EXPERIMENTATION USING GA}


In the present work, GA is used for solving different size of flow shop scheduling problems. The tested levels of jobs range from 5 to 100 jobs, while those of machines range between 4 and 20 machines. Two different ranges of processing time variability and five different random seeds are used to generate the flow-shop scheduling problems. Processing times are uniformly distributed as follows:

Set I Problems: Processing times range between $(6,30)$

Set II Problems: Processing times range between $(20,50)$.

The different sizes of problems considered are shown in Table 1.

\begin{tabular}{|c|c|c|}
\hline Sl. No. & Number of Jobs, N & Number of Machines, M \\
\hline 1 & 5 & 4 \\
\hline 2 & 10 & 5 \\
\hline 3 & 15 & 5 \\
\hline 4 & 20 & 5 \\
\hline 5 & 25 & 10 \\
\hline 6 & 30 & 10 \\
\hline 7 & 40 & 10 \\
\hline 8 & 30 & 15 \\
\hline 9 & 40 & 15 \\
\hline 10 & 50 & 10 \\
\hline 11 & 50 & 20 \\
\hline 12 & 80 & 10 \\
\hline 13 & 80 & 20 \\
\hline 14 & 100 & 10 \\
\hline 15 & 100 & 20 \\
\hline \multicolumn{2}{|c|}{ Table 1 Problems Considered } \\
\hline
\end{tabular}

The parameters used for GA are as follows.

- Population size

$$
\begin{aligned}
& 80 \text { - Up to } 30 \text { jobs } \\
& 150 \text { - More than } 30 \text { jobs }
\end{aligned}
$$

- Crossover probability -0.6

- Mutation probability $\quad-0.1$

- Number of generations $\quad-1000$

The number of generations is chosen as 1000 based on a pilot study which indicates that GA converges at this number of generations. The data for the pilot study are as follows. Problem Size: 30 Jobs 15 Machines; Parameters of GA: Cross over probability - 0.6; Mutation Probability - 0.1; Population Size - 80. Two-point cross over with shift mutation. Figure 3 shows the convergence result. 


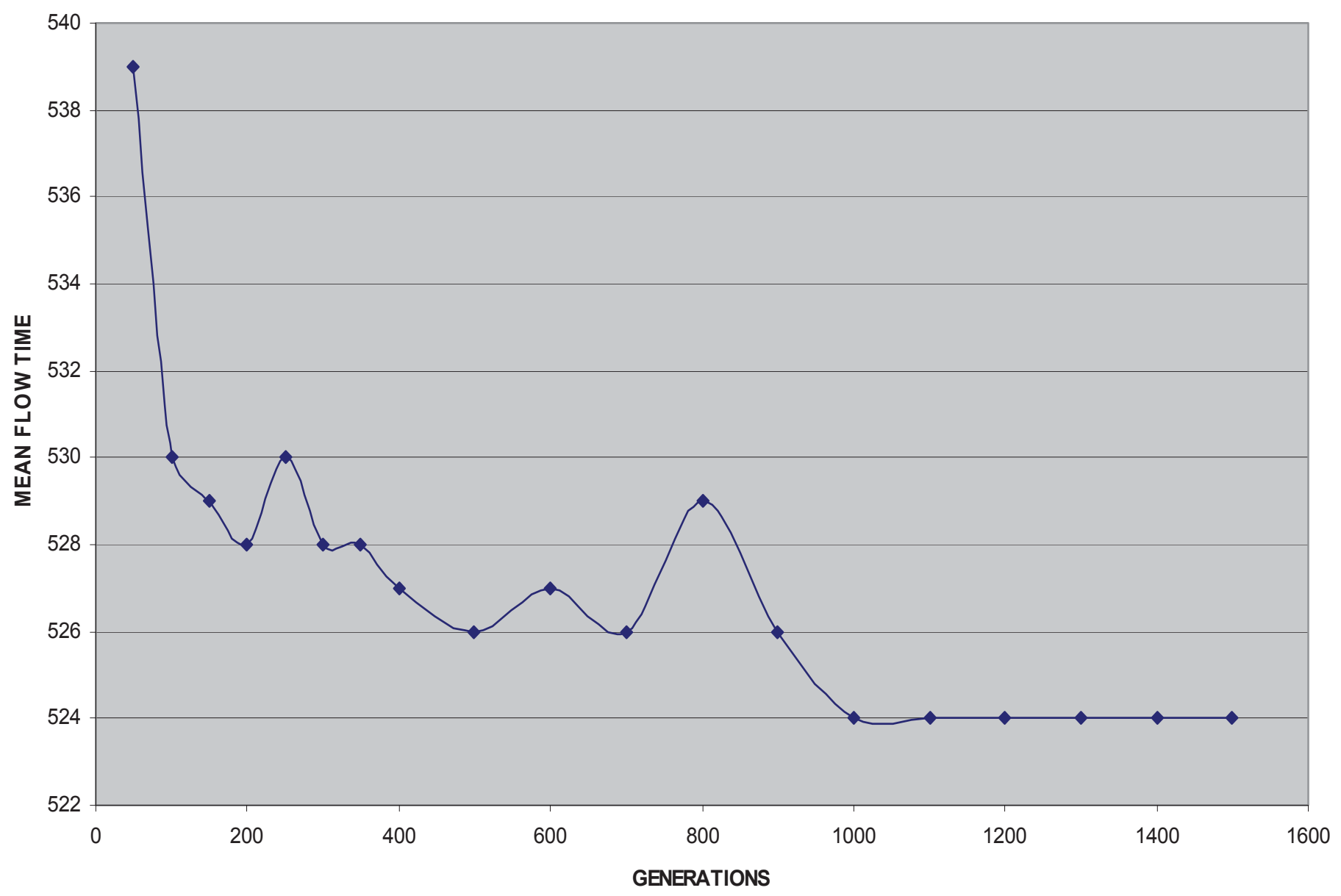

\section{RESULTS AND DISCUSSION}

The Table 2 and Table 3 present the mean flow time values for the set - I and Set - II problems of various sizes respectively when single point crossover and two point crossover operators are used in GA. For each of the crossover operators, shift mutation and random exchange mutation are also used. These results reveal that two point crossover with shift mutation gives better mean flow time values for many problems, especially for large size problems. However, for small size problems, there is no generalization possible regarding the crossover and mutation operations. 


\begin{tabular}{|c|c|c|c|c|c|}
\hline \multirow{2}{*}{ S.NO } & \multirow{2}{*}{$\mathbf{N} \mathbf{X} \mathbf{M}$} & \multicolumn{2}{|c|}{ SINGLE POINT CROSSOVER } & \multicolumn{2}{|c|}{ TWO POINT CROSSOVER } \\
\hline & & $\begin{array}{c}\text { SHIFT } \\
\text { MUTATION }\end{array}$ & $\begin{array}{c}\text { RANDOM } \\
\text { EXCHANGE }\end{array}$ & $\begin{array}{c}\text { SHIFT } \\
\text { MUTATION }\end{array}$ & $\begin{array}{c}\text { RANDOM } \\
\text { EXCHANGE }\end{array}$ \\
\hline 1 & $5 \times 4$ & 104 & 104 & 104 & 104 \\
\hline 2 & $10 \times 5$ & 164 & 165 & 165 & 165 \\
\hline 3 & $15 \times 5$ & 209 & 206 & 205 & 205 \\
\hline 4 & $20 \times 5$ & 248 & 246 & 246 & 249 \\
\hline 5 & $25 \times 10$ & 401 & 404 & 404 & 405 \\
\hline 6 & $30 \times 10$ & 430 & 406 & 443 & 406 \\
\hline 7 & $40 \times 10$ & 540 & 520 & 494 & 528 \\
\hline 8 & $50 \times 10$ & 676 & 660 & 623 & 670 \\
\hline 9 & $30 \times 15$ & 538 & 521 & 518 & 519 \\
\hline 10 & $40 \times 15$ & 679 & 615 & 622 & 645 \\
\hline 11 & $50 \times 20$ & 919 & 905 & 892 & 911 \\
\hline 12 & $80 \times 10$ & 970 & 970 & 968 & 965 \\
\hline 13 & $80 \times 20$ & 1251 & 1248 & 1244 & 1244 \\
\hline 14 & $100 \times 10$ & 1174 & 1162 & 1162 & 1163 \\
\hline 15 & $100 \times 20$ & 1463 & 1458 & 1455 & 1458 \\
\hline
\end{tabular}

Table 2 Mean Flow Time for Set - I Problems with GA 


\begin{tabular}{|c|c|c|c|c|c|}
\hline \multirow{2}{*}{ S.NO } & \multirow{2}{*}{ N X M } & \multicolumn{2}{|c|}{$\begin{array}{l}\text { SINGLE POINT } \\
\text { CROSSOVER }\end{array}$} & \multicolumn{2}{|c|}{ TWO POINT CROSSOVER } \\
\hline & & $\begin{array}{c}\text { SHIFT } \\
\text { MUTATION }\end{array}$ & $\begin{array}{c}\text { RANDOM } \\
\text { EXCHANGE }\end{array}$ & $\begin{array}{c}\text { SHIFT } \\
\text { MUTATION }\end{array}$ & $\begin{array}{c}\text { RANDOM } \\
\text { EXCHANGE }\end{array}$ \\
\hline 1 & $5 \times 4$ & 208 & 205 & 208 & 204 \\
\hline 2 & $10 \times 5$ & 325 & 325 & 324 & 326 \\
\hline 3 & $15 \times 5$ & 405 & 406 & 404 & 406 \\
\hline 4 & $20 \times 5$ & 487 & 489 & 486 & 493 \\
\hline 5 & $25 \times 10$ & 776 & 776 & 771 & 779 \\
\hline 6 & $30 \times 10$ & 830 & 816 & 857 & 803 \\
\hline 7 & $40 \times 10$ & 1041 & 1007 & 981 & 1014 \\
\hline 8 & $50 \times 10$ & 1040 & 1022 & 1039 & 1023 \\
\hline 9 & $30 \times 15$ & 1275 & 1222 & 1205 & 1238 \\
\hline 10 & $40 \times 15$ & 1266 & 1250 & 1226 & 1270 \\
\hline 11 & $50 \times 20$ & 1701 & 1706 & 1666 & 1703 \\
\hline 12 & $80 \times 10$ & 1842 & 1844 & 1820 & 1810 \\
\hline 13 & $80 \times 20$ & 2308 & 2310 & 2306 & 2307 \\
\hline 14 & $100 \times 10$ & 2210 & 2211 & 2206 & 2207 \\
\hline 15 & $100 \times 20$ & 2704 & 2700 & 2694 & 2697 \\
\hline
\end{tabular}

Table 3 Mean Flow Time for Set II Problems with GA 


\section{CONCLUSION}

In the present study, GA is applied to solve the flow shop scheduling problem. Different sizes of problems are considered for analysis. In implementing GA, single-point cross over, two-point cross over, shift mutation and random exchange mutation are used. Two sets of problems with respect to the processing times are considered. The results reveal that two point crossover with shift mutation provides better mean flow time values for large size problems. The present study can be extended by improving GA. This improvement can be made by using one solution in the initial population as the solution obtained from an existing heuristic and the remaining generated randomly. The results obtained using GA can also be compared with that obtained by using other heuristics. The effect of some other combination of crossover and mutation operators on GA can be studied.

\section{REFERENCES}

[1] French, S, Sequencing and Scheduling: An Introduction to the mathematics of the job-shop, Ellis Horwood Series in Mathematics and Its Applications, ed. G.M. Bell (Chichester: Ellis Hollwood Limited, 1982).

[2] Baker, K. R., Introduction to Sequencing and scheduling, John Wiley \& Sons, Inc., 1974.

[3] Framinan,J.M, Leisten.R, Ruiz-Usano.R,. Comparison of heuristics for flow time minimisation in permutation flowshops. Computers \& Operations Research 32 (2005) 1237-1254.

[4] Nawaz, M., Enscore Jr. E., and ham, I., A heuristic algorithm for the 'm' machine,'n' job flow shop sequencing problem, OMEGA, Vol. 11, No. 1, 91-95,1983.

[5] Pannerselvam, R., Production and operations management, Prentice Hall of India Pvt.Ltd, 1999.

[6] Reeves, C.R., A genetic algorithm for flow shop sequencing, Computers Ops Res. Vol. 22, No. 1, 5-13, and 1995

[7] Goldberg, D.E., Genetic Algorithms in Search, Optimization, and Machine Learning. Addison-Wesley, Reading, 2000. 